\title{
Peculiarities of admittance spectroscopy study of wide bandgap semiconductors on the example of diamond
}

\author{
$A V$ Solomnikova ${ }^{1}, V A$ Lukashkin $^{1, *}$, and $O V$ Derevianko $^{2}$ \\ ${ }^{1}$ St. Petersburg State Electrotechnical University (LETI), Professor Popov Street 5, 197376 St. Petersburg, Russia \\ ${ }^{2}$ Peter the Great Saint-Petersburg, Polytechnic University, Polytechnicheskaya 29, 194064, Russian Federation
}

\begin{abstract}
To improve the performance characteristics of power and high-frequency electronics, wide bandgap semiconductors are now widely used. This paper presents consideration of features arising during exploration of electronic characteristics of wide bandgap materials. We use the admittance spectroscopy method for analyzing free carrier concentration and boron-impurity activation energy in semiconductor diamond. The special aspect that should be taken into account while studying wide bandgap materials is incomplete ionization of impurity. In this work we adjust the experimental conditions, basing on the previous experience, in particular reduce signal/noise ratio and reckon with heat capacity of the samples and substrate. As a result we obtained high quality conductance spectra and activation energy of boron impurity in low-doped diamond.
\end{abstract}

\section{Introduction}

Presently, the trend in electronic devices for power and high frequency application is to shift to wide bandgap semiconductor materials [1-4]. This shift is caused by the performance threshold achieved by classical semiconductors such as $\mathrm{Si}, \mathrm{Ge}$ and GaAs. The most popular parameters which are used for comparison of materials for high performance electronics are: Baliga figure of merit [5], Johnson figure of merit [6], Keyes figure of merit [7]. The numerical values of these figures of merit for the most common wide bandgap semiconductors are presented in Table 1 (the values for Si are taken equal to 1 , and the others are normalized by it).

As it is seen from Table 1, one can expect to achieve the best performance characteristics for the devices based on semiconductor diamond. It is an ultimate material due to its giant package density and large bandgap, and being doped to achieve semiconductor properties it is a perfect match for power and highfrequencies electronics [10-12].

The most important parameter, which governs the performance of semiconductor devices, is free carrier concentration. Such devices show the best characteristics are achieved at total ionization of impurity. Si and similar materials reach complete ionization at room temperature. In contrast to them, ionization at room temperature for the above-mentioned diamond and $\mathrm{SiC}$, is $0.2 \%$ and $25 \%$, respectively. That is why during the experiments to obtain concentration profiles and activation energy of the doping impurity of wide bandgap materials, it is necessary to alter the experimental conditions to obtain reliable results.

The aim of this work was to obtain activation energy of boron impurity in diamond from frequency and temperature admittance spectra. To carry out these measurements we had to adjust the experimental conditions.

\section{Materials and methods}

In this work, we used admittance spectroscopy technique to study the electrical properties of semiconductor diamond plates doped by boron. The plates were cut from single-crystal diamond, synthesized by high pressure high temperature (HPHT) method. The samples

Table 1. Comparison of figures of merit normalized by Si for diamond, $4 \mathrm{H}-\mathrm{SiC}$, and $\mathrm{GaN}[8,9]$.

\begin{tabular}{|c|c|c|c|c|}
\hline Characteristics & Diamond & 4H-SiC & GaN & Si \\
\hline $\begin{array}{c}\text { Baliga figure } \\
\text { of merit (BFOM) }\end{array}$ & 474 & 5.1 & 10.6 & 1 \\
\hline $\begin{array}{c}\text { Jonson figure } \\
\text { of merit (JFOM) }\end{array}$ & 6009 & 1217 & 2347 & 1 \\
\hline $\begin{array}{c}\text { Keyes figure } \\
\text { of merit (KFOM) }\end{array}$ & 35 & 6.8 & 1.6 & 1 \\
\hline
\end{tabular}

\footnotetext{
* Corresponding author: lukashkinv@gmail.com
} 

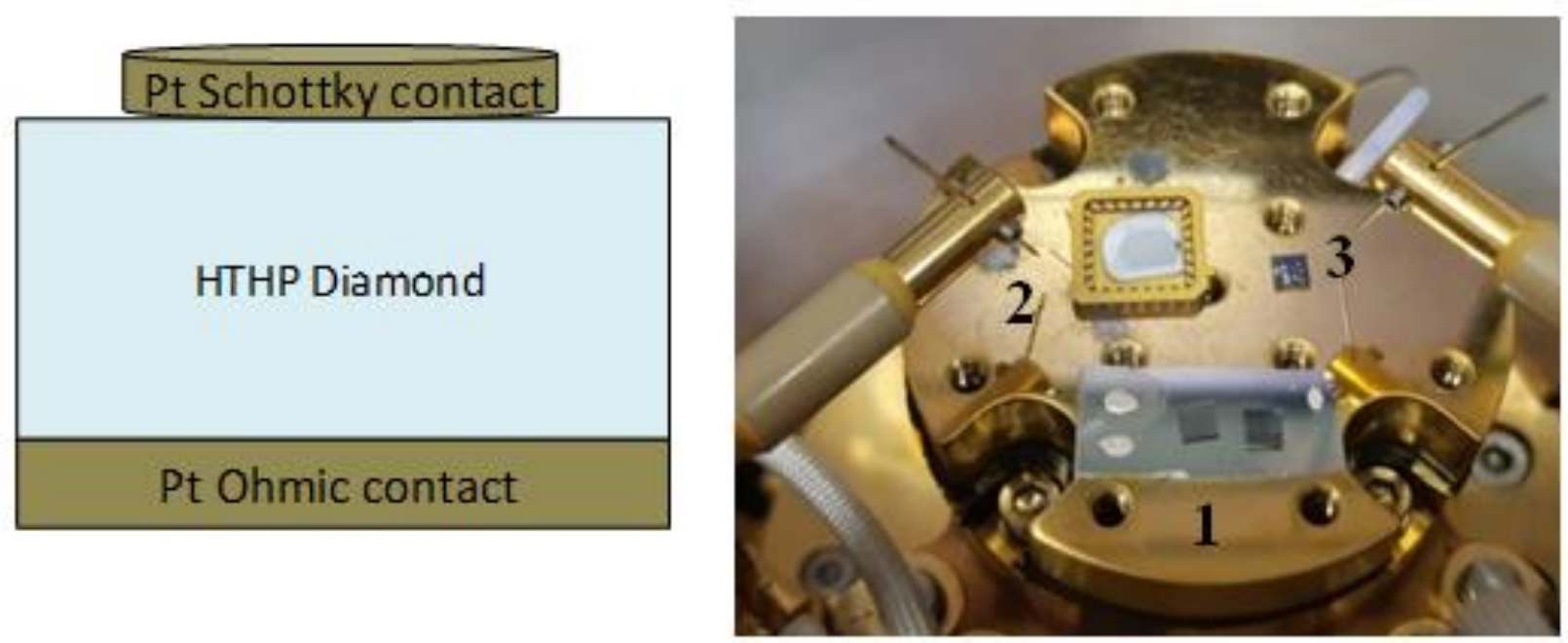

Fig. 1. a) Structure of the samples being investigated; b) Samples placement inside the cryogenic probe station chamber.

had multisectorial structure, i.e. within one plate there were areas of various boron concentration: low-doped areas were of white color, heavily-doped areas were of blue color. According to preliminary FTIR assessment of the boron content, the boron concentration in these areas differed for at least 10 times.

Contacts were formed on the opposite side of the diamond samples. The upper side contained an array of round Platinum contacts with $130 \mu \mathrm{m}$ diameter. The thickness was $100 \mathrm{~nm}$, these contacts were obtained at $300{ }^{\circ} \mathrm{C}$. Bottom side was completely covered with platinum to make an Ohmic contact of the same thickness as Schottky contact. Ohmic contact was deposited at $70{ }^{\circ} \mathrm{C}$ (figure 1a).

There are a lot of techniques used for diagnosing the electronic properties of the semiconductor materials. Admittance spectroscopy is one of the most useful methods [13], figure 2. A huge variety of electrical characteristics can be obtained by this method, for example charge carrier concentration, activation energy of impurities, which gives relevant information about samples.

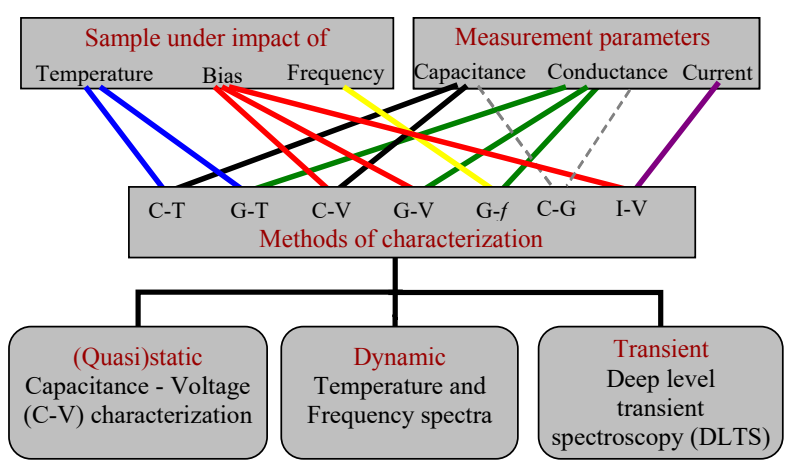

Fig. 2. Schematic representation of admittance methods tree.

Depending on the purpose of the study, the characteristics of the structure being measured and the properties of impurity centers admittance spectroscopy allow one to implement quasistatic, dynamic and non- stationary methods. Dynamic admittance spectroscopy methods allow obtaining temperature conductance spectra at a given set of frequencies and frequency conduction spectra at selected temperatures.

Admittance measurements were carried out by means of computer-controlled setup, the main parts of which are RLC-meter Agilent E4980A, closed-cycle helium probe station Janis CCR-10, and temperature controller LakeShore 336. The setup covers a temperature range 20 $-450{ }^{\circ} \mathrm{C}$ and frequency range $1 \mathrm{kHz}-2 \mathrm{MHz}$ with capacitance resolution up to $1 \mathrm{fF}$.

Figure $1 \mathrm{~b}$ presents the sample chamber of Janis CCR-10-2 probe station with samples placed on the golden object table. As it can be seen from figure $1 \mathrm{~b}$, samples can be placed on sitall substrate (figure $1 b ; 1$ ), ceramic cell (figure $1 b ; 2$ ) and directly on the object table (figure $1 \mathrm{~b} ; 3$ ).

To create a closed system inside the probe station we decided to put the sample on the cell. It was done to withdraw the induced noise. Sitall wafer can also eliminate the induced signal, but it has a higher thermal capacity than the sample. This can cause an error, which can be hardly identified.

Further we give some theoretical background on the admittance spectroscopy investigation technique, and the more complex description is presented in [13, 14]. A deep trap like boron in single crystal diamond can be characterized by emission rate of charge carriers to the corresponding band (valence band in this case)

$$
e_{p}=g_{A} t_{t h} \sigma_{p} N_{V} \exp \left(-E_{a} / k T\right)
$$

where $N_{V}$ is the effective density of states, $v_{t h}$ is the thermal velocity for holes, $\sigma_{p}$ is the hole capture cross section, and $g_{A}$ is the degeneracy factor for the acceptor level.

Taking in mind the temperature dependence of the pre-exponential factors, one usually rewrites (1) in the form 


$$
e_{p}=A T^{2} \exp \left(-E_{a} / k T\right),
$$

where factor $A$ is independent on temperature.

When a small ac signal of frequency $\omega$ is applied to the sample with fabricated Schottky and Ohmic contacts, a trap with the emission rate (1) generates a conductance (and capacitance as well) response of the form

$$
G(T)=\frac{e_{p}(T) \omega^{2}}{e_{p}^{2}(T)+\omega^{2}} \frac{N_{T}}{p} S\left(\frac{\varepsilon \varepsilon_{0} e N_{A}^{-}}{2 V}\right)^{1 / 2},
$$

where $N_{T}$ is the trap concentration, and $p$ is the free charge carrier concentration.

\section{Results and discussions}

\subsection{Experiment parameters set-up}

To obtain reliable data first of all we should eliminate possible experimental error, also it should be critically analyzed from physical standpoint.

It is known that experimental errors, in general case, could be divided into [15]:

- $\quad$ Systematic (those that do not change at multiple experiment repeating), i.e. fault in adjustment of a "zero" level;

- $\quad$ Random (which are caused by a large number of factors, which cannot be taken into account), i.e. perturbance in electrical supply, accuracy of an equipment, induced signal in wire;

- Gross (a measurement, which goes far beyond the range of a random error), i.e. caused by disturbance of voltage, defect in equipment.

The main goal of our study is precise definition of activation energy of boron impurity in diamond. During investigations by admittance spectroscopy, it becomes necessary to pay extra attention to signal/noise ratio (random error) and temperature definition (systematic error) as the main errors of such experiments. That limits the accuracy of the determination of the activation energy. Further we will describe the listed errors and ways to eliminate them.

\subsubsection{Test signal amplitude}

First of all we considered frequency conductance spectra obtained by fixing the temperature and measuring the conductance at various test signal frequencies in the range from $500 \mathrm{~Hz}$ to $2 \mathrm{MHz}$.

Comparison of conductance spectra at different test signal levels $(30 \mathrm{mV}$ and $80 \mathrm{mV})$ obtained at room temperature for the low-doped sample area is presented in figure 3.



Fig. 3. Frequency spectra at the different test signal amplitude in the low doping area.

Taking into account that the low-doped (white) area contains a small amount of free charge carriers, the conductance response from it is quite low. The measurements were carried out at the edge of equipment resolution. So, obtaining the high-quality spectra is becoming quite a difficult task. The increasing of signal amplitude from 30 to $80 \mathrm{meV}$ leads to smothering of the spectrum shape which is driven by the necessity to obtain a free charge carrier from larger area within the low dopant semiconductor. Thus, error, connected with signal/noise ratio, can be eliminated. Besides, we should emphasize that this method of increasing signal/noise ratio can be used only in bulk semiconductor without thin structures. So, in out further measurements we used the test signal of $80 \mathrm{meV}$.

\subsubsection{Accuracy of temperature determination}

For temperature evaluation there are five temperature sensors inside the probe station, and two of them are located inside the chamber. Temperature inside the chamber is controlled by Lakeshore 336 temperature controller. This controller allows one to preset any temperature change speed. LakeShore 336 has two different systems, which regulate temperature alteration: Proportional-Integral-Derivative (PID) and linear regulation. The latter is described by factor of proportionality, called "Ramp Rate". Ramp Rate $=4$ was set at the test launch, which is equivalent to temperature change speed of $4 \mathrm{~K} / \mathrm{min}$. Temperature conductance spectra at this Ramp Rate, with falling and rising temperature were obtained. These spectra show significant hysteresis which is unacceptable. Hysteresis can critically influence the experiment results.

Further experiments were conducted with temperature change rate $\approx 0.5 \mathrm{~K} / \mathrm{min}$. We see that the mismatch of the conductance spectra is become infinitesimal. Thus, when temperature rate is chosen below $0.5 \mathrm{~K} / \mathrm{min}$, the obtained spectra are of high quality and repeatability.

The other issue is to adjust temperature while recording frequency conductance spectra. When we set the desired temperature, thermal capacity of samples and substrate have an influence on time interval, needed for reaching the setpoint and, therefore, the true spectrum shape can be disturbed. To test this, a set of experiments 
with different time interval were conducted. The reference temperature point was $240 \mathrm{~K}$, as it allowed us to catch the conductance response for both areas of sample with various concentrations. Below we present graph that shows frequency spectra at different thermostating time (figure 4).



Fig. 4. Variation of frequency spectra at different thermostating times.

The example presented in figure 4 shows that without proper temperature stabilizing, a shift of conductance peak from $510 \mathrm{kHz}$ to $740 \mathrm{kHz}$ is obtained. It is a quite great error, approximately $20 \%$. This error results in a drastic change in the derived activation energy up to 70 $\mathrm{meV}$ : not the expected $320 \mathrm{meV}$, which correspond to boron impurity activation energy in the low-doped area of diamond, but $390 \mathrm{meV}$. Thermostating for 15 and 30 minutes gives the same spectra, as it can be seen from figure 4. So, we can conclude that temperature stabilization for more than $15 \mathrm{~min}$ is excessive, and further temperature stabilizing does not improve the accuracy in spectra obtaining, but slows the experiments and increasing research cost. Therefore the 15 minutes thermostating for obtaining G-f spectra is optimum and we will use it in our experiments.

\subsection{The obtained spectra and activation energies}

The obtained thermal conductance spectra and calculated activation energy are shown below, figure 5 .

The obtained thermal conductance spectra at various frequencies and the constructed Arrhenius plot are shown in Figure 5. Activation energy, calculated from temperature conductance spectra, is $293 \pm 2 \mathrm{meV}$, which corresponds to boron impurity in heavily-doped diamond [16]. The mean squared error (MSE) is $2 \mathrm{meV}$, which is less than $1 \%$. High precision of the calculated activation energy is due to high quality of obtained temperature spectra and careful adjustment of experimental parameters.

\section{Conclusions}

Investigations of the wide bandgap materials, such as diamond, are associated with some specific features attributed to these semiconductors. In this paper we present and analyze special aspects which should be addressed during admittance spectroscopy experiments. The systematization of the errors as well as their influence on the obtained data accuracy and repeatability are described. During the research, both thermal and frequency conductance spectra were obtained with high accuracy. The small MSE for the calculated activation energy of boron in low-doped diamond proves that the experimental parameters were carefully adjusted.
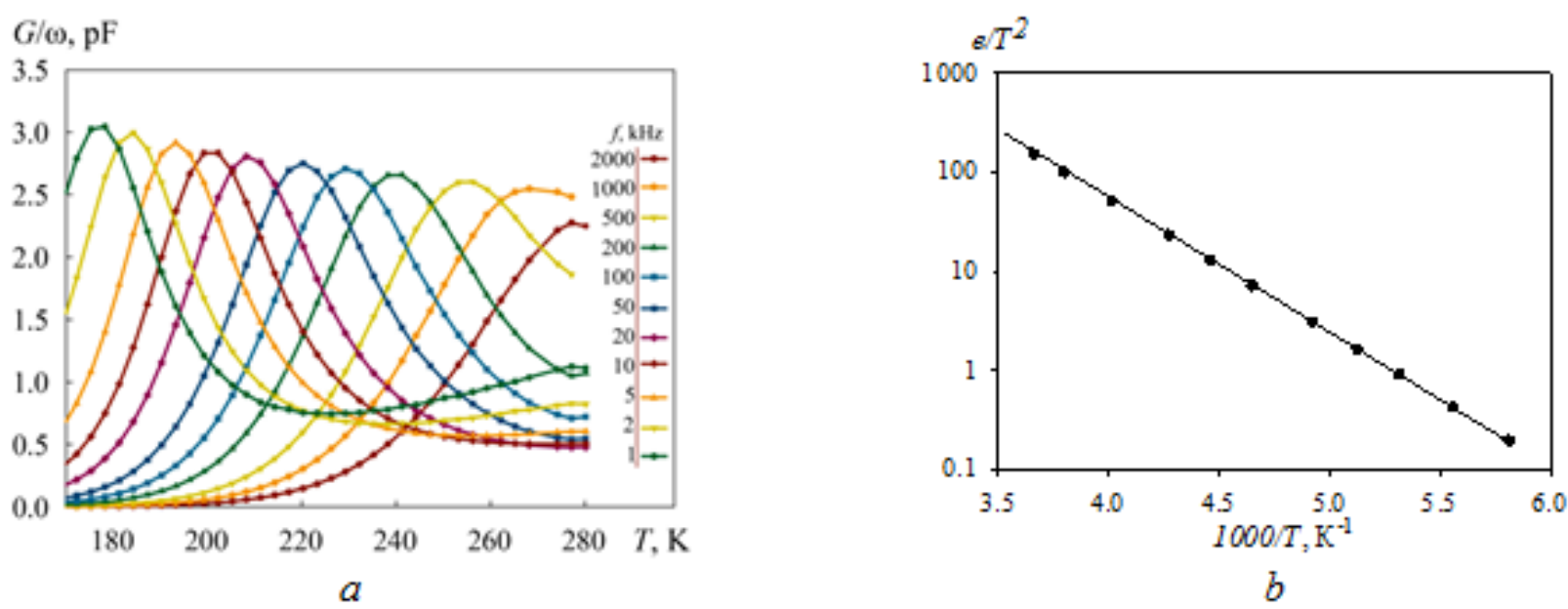

Fig. 5. Temperature admittance spectra (a) and Arrhenius plot (b) for the blue area of the sample. 


\section{References}

[1] E.Kohn, A.Denisenko, Thin Solid Films, 515, 4333-4339 (2007).

[2] V.Emtsev, E. Gushchina, V. Petrov, N.Tal'nishnih, A.Chernyakov, E. Shabunina, N. Shmidt, A. Usikov, A. Kartashova, A. Zybin, V. Kozlovski, M. Kudoyarov, A. Saharov, A. Oganesyan, D. Poloskin, V.Lundin, Semiconductors, 52, 942-949 (2018).

[3] S. Petrov, A. Alexeev, V. Mamaev, D. Krasovitsky, V. Chaly, Proceedings of SPIE The International Society for Optical Engineering, 10224 (2016).

[4] A. Lebedev, E. Kalinina,V. Kozlovski, Journal of Surface Investigation, 12, 2, 364-369 (2018).

[5] B. Baliga, IEEE Electron Device Letters, 10, 10, 455-457 (1989).

[6] E. Johnson, RCA Rev., 163-177 (1965).

[7] R. Keyes, 1972 Proc. IEEE, 60, 225 (1972).

[8] C. Wort, R. Balmer, Materials Today,11, 1-2, 2228 (2008).

[9] NSM Archive - Physical Properties of Semiconductors [Electronic available]. Available at: http://www.ioffe.ru/SVA/NSM/.

[10] A. Huang, IEEE Electron Device Letters, 25, 5, 298-301 (2004).

[11] J. Achard,F.Silva, R. Issaoui, O. Brinza, A. Tallaire, H. Schneider, K. Isoird, H. Ding, S. Koné, M. Pinault, F. Jomard, A. Gicquel, Diamond and Related Materials, 20, 2, 145-152 (2011)

[12] V. Blank, V. Bormashov, S. Tarelkin, S. Buga, M. Kuznetsov, D. Teteruk, N. Kornilov, S. Terentiev, A. Volkov, Diamond and Related Materials, 57, 32-36 (2015).

[13] V. Zubkov, O. Kucherova, S. Bogdanov, A. Zubkova, J. Butler, V. Ilyin, A. Afanas'ev, A. Vikharev, Journal of Applied Physics, 118, 145703 (2015).

[14] O.V. Kucherova, V.I. Zubkov, E.O. Tsvelev, I.N. Yakovlev, A.V. Solomonov, Inorganic Materials, 47, 1574 (2011).

[15] V. Zubkov, Computer technologies in research studies, (Saint-Petersburg: Publishing House of SPbGETU "LETI", 2010) (in Russian).

[16] V. Zubkov, A. Solomnikova, J. Post, E. Gaillou, J. Butler, Diamond and Related Materials, 72, 8793 (2017). 